\title{
Image of the month: A case of phlebosclerotic colitis
}

\author{
Authors: Ying-Kuo Liu, ${ }^{A}$ Ming-Hai Du ${ }^{B}$ and Yuan-Pin Hsu ${ }^{\mathrm{C}, \mathrm{D}, \mathrm{E}}$
}

\section{Case presentation}

A 74-year-old man presented with recurrent right lower quadrant abdominal pain and diarrhoea for many years. He had no systemic disease. However, he had taken Chinese herbs for more than 50 years. Physical examination revealed rightsided abdomen tenderness. Laboratory examination showed his white cell count to be $3,680 / \mu \mathrm{L}$ and $\mathrm{C}$-reactive protein of $4.6 \mathrm{mg} / \mathrm{dL}$. Heavy metal testing results were within the normal range. Plain radiograph revealed serpiginous calcifications over the upper-right quadrant of the abdomen (Fig 1a). Computed tomography (CT) showed dendritic calcifications along the superior mesenteric vein and wall thickness of ascending and transverse colon (Fig 1b). Colonoscopy with biopsy demonstrated fibrous thickening, sclerosis in submucosa and dilated tortuous veins. Operation was suggested, but the patient refused. The patient continued to be bothered by recurrent symptoms and hospitalised three times during a follow-up period of 1 year.

Phlebosclerotic colitis (PC) is usually found in Asian or Asian immigrants in Western countries. ${ }^{1}$ PC is characterised by impairing venous return from the colon due to sclerosis and fibrosis of mesenteric veins, resulting in chronic ischemia, intestinal

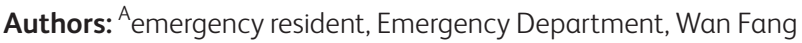
Hospital, Taipei Medical University, Taipei, Taiwan; ${ }^{B}$ emergency physician, Emergency Department, Wan Fang Hospital, Taipei Medical University, Taipei, Taiwan; ' emergency physician, Emergency Department, Wan Fang Hospital, Taipei Medical University, Taipei, Taiwan; Demergency physician, Department of Emergency, School of Medicine, College of Medicine, Taipei Medical University, Taipei, Taiwan; 'Emaster's student, Graduate Institute of Clinical Medicine, College of Medicine, Taipei Medical University, Taipei, Taiwan

obstruction, and even perforation. ${ }^{2}$ The etiology and pathogenesis remains unclear but may be related to long-term use of Chinese herbs, alcohol abuse or ingestion of other toxic materials. ${ }^{1-3}$

Diagnosis of $\mathrm{PC}$ is often delayed due to nonspecific clinical symptoms. Abdominal radiograph and CT sharing characteristic radiologic findings in common with multiple fine, tortuous, serpentine calcifications along mesenteric vessels and the involved colon play an important role. ${ }^{1-4} \mathrm{CT}$ provides additional information about complications and colon wall thickening, especially in the right colon. Colonoscopy findings, including dark purple discolorations of the mucosa with mucosal edema and erythema, erosions, or ulcerations, are used to verify the diagnosis. ${ }^{1}$ There is no consensus on treatment. Total colectomy or subtotal colectomy is the mainstay of treatment in most cases in the literature. However, Yu et al recommend conservative treatment for initial mild cases. ${ }^{4}$.

\section{Author Contributions}

YKL reviewed the case and wrote the manuscript. MHD contributed to the case and manuscript revision. YPH contributed to manuscript review. 


\section{Consent for publication}

Consent was obtained from the patient to publish the clinical details and images from this case.

\section{References}

1 Lee SM, Seo JW. Phlebosclerotic colitis: case report and literature review focused on the radiologic findings in relation to the intake period of toxic material. Jpn J Radiol 2015;33:663-7.

2 Lo WK, Mahboobani NR, Siu Y. Gastrointestinal: Phlebosclerotic colitis: A rare but increasingly recognized cause of ischemic colitis with telltale imaging features. J Gastroenterol Hepatol 2017;32:1792.
3 Hu P, Deng L. Phlebosclerotic colitis: three cases and literature review. Abdom Imaging 2013;38:1220-4.

4 Yu CJ, Wang HH, Chou JW et al. Phlebosclerotic colitis with nonsurgical treatment. Int J Colorectal Dis 2009;24:1241-2.

Address for correspondence: Dr Yuan-Pin Hsu, Department of Emergency and Critical Care Medicine, Wan Fang Hospital, Taipei Medical University, No. 111, Sec. 3, Xinglong Road, Wenshan District, Taipei City 116, Taiwan.

Email: koakoahsu@gmail.com lively tour of colourful characters and dubious cures that have littered the Royal College of Physicians' 500-year history.

Order by phone or online

$\mathrm{Tel}+44(0) 2030751358$

or visit www.rcplondon.ac.uk/shop
Price: $€ 12$

(Postage and packaging not included)

$10 \%$ discount for fellows and members,

$15 \%$ discount for foundation doctors and students 